\title{
Review of "Diamond Open Access"
}

Robert West $^{1}$

1 Univers ity College London, University of London

This is a very useful entity to define and the definition is clear and simple. It may be useful to add subclasses representing different variants, such as the one adopted by Qeios. 